\title{
Femoral Revision Hip Arthroplasty
}

\section{A Comparison of Two Stem Designs}

\author{
Corey J. Richards MD, MASc, FRCSC, \\ Clive P. Duncan MD, MSc, FRCSC, Bassam A. Masri MD, FRCSC, \\ Donald S. Garbuz MD, MHSc, FRCSC
}

Published online: 22 October 2009

(C) The Association of Bone and Joint Surgeons (B) 2009

\begin{abstract}
For various reasons the tapered, fluted, modular titanium (TFMT) stem has become our component of choice over cylindrical, nonmodular cobalt chrome (CNCC) components for THA revision. We therefore asked whether the TFMT femoral components better achieved three important goals of revision arthroplasty than CNCC stems: (1) improving quality of life; (2) avoiding complications; and (3) preserving or restoring femoral bone stock. We compared patients undergoing femoral component revision hip arthroplasty with either a CNCC $(\mathrm{N}=105)$ component or a TFMT $(\mathrm{N}=95)$ component to determine if the increased use of TFMT components is justified. We retrospectively reviewed all patients undergoing revision total hip arthroplasty between January 2000 and March 2006. All eligible patients completed outcome questionnaires (WOMAC, SF-12, Oxford-12, UCLA Activity Score, and Satisfaction Scores). Radiographs were evaluated for loosening and preservation or restoration of the proximal femur host bone. The TFMT and CNCC
\end{abstract}

Each author certifies that he or she has no commercial associations (eg, consultancies, stock ownership, equity interest, patent/licensing arrangements, etc) that might pose a conflict of interest in connection with the submitted article.

Each author certifies that his or her institution approved the human protocol for this investigation, that all investigations were conducted in conformity with ethical principles of research, and that informed consent for participation in the study was obtained.

\section{J. Richards, D. S. Garbuz ( $₫)$}

Division of Lower Limb Reconstruction and Oncology, Department of Orthopaedics, University of British Columbia, 3114-910 West 10th Avenue, Vancouver, BC V5Z 4E3, Canada e-mail: donald.garbuz@vch.ca; garbuz@shaw.ca

C. P. Duncan, B. A. Masri

Department of Orthopaedics, University of British Columbia,

Vancouver, BC, Canada cohorts were comparable with respect to age, gender, diagnosis, and comorbidities. The TFMT cohort had worse preoperative bone defects (65\% Paprosky 3B and 4). The TFMT cohort had higher outcome scores (WOMAC pain, WOMAC stiffness, Oxford-12, and Satisfaction), fewer intraoperative fractures, and better restoration of the proximal femur host bone. Our data suggest the TFMT stem provided improved clinical outcomes (improved quality of life, decreased complications, and preservation of bone stock) than the CNCC stem.

Level of Evidence: Level III, therapeutic study. See Guidelines for Authors for a complete description of levels of evidence.

\section{Introduction}

Failure of the femoral component after a primary or revision THA is commonly associated with some degree of femoral bone loss. Depending on the quantity and quality of the remaining host bone, femoral stem revision can be challenging. Four important goals of reconstructing failed femoral components include achieving long-term implant fixation, improving patient quality of life, avoiding complications, and maintaining or restoring proximal femoral host bone stock.

Cylindrical, nonmodular, cobalt chromium (CNCC) stems have been commonly used for cementless femoral component revision in North America for some years [13, 16, 19, 23]. Although long-term survivorship has been well established (95\%-96\% survivorship at a mean of 10 14 years [13, 23]), concerns have been raised regarding postoperative thigh pain $(8 \%-30 \%[16,19])$, a high incidence of intraoperative fractures $(9 \%-30 \%[18,23])$, and stress shielding of the proximal femur (reportedly 8\% [19]) 
with the use of this stem design. Several femoral component designs, with varying degrees of modularity and different fixation concepts, have been developed in an attempt to address these concerns. Many of these designs have achieved comparable levels of implant survivorship (95\%-99\% survivorship at 7-14 years [3-5].

Tapered, fluted, modular, titanium (TFMT) femoral components have been used internationally $[2,3]$ but have only recently been introduced in North America as an alternative revision stem design. These stems reportedly have high early survivorship (95\%-98\%) at a mean of 24.2 years $[17,20]$. The increased modularity, decreased stiffness, and tapered stem geometry of this new design may enable surgeons to achieve the previously mentioned goals of femoral component revision. In a study of 220 patients we previously reported that quality of life for 34 patients revised with a TFMT femoral component was better (Oxford hip score 79 (TFMT) versus 69 (CNCC), $\mathrm{p}=0.006$; Satisfaction score 97 (TFMT) versus 83 (CNCC), $\mathrm{p}=0.0001$; WOMAC function 75 (TFMT) versus $69(\mathrm{CNCC}), \mathrm{p}=0.02$; WOMAC pain 84 (TFMT) versus $75(\mathrm{CNCC}), \mathrm{p}=0.03$ ) than a cohort of 186 patients receiving CNCC stems [11]. Modularity facilitates the intraoperative adjustment of leg length, horizontal offset, and neck version. Enhancing hip biomechanics may result in better patient function, improved quality of life as well as a decreased risk of postoperative instability. Titanium has a lower modulus of elasticity when compared with cobalt chromium, resulting in reduced femoral component stiffness for an equivalent diameter stem. By reducing the modulus mismatch between the femoral component and the host bone, titanium stems may result in less thigh pain and less proximal femoral stress shielding, particularly for smaller stem diameters. Because stiffness is a function of the radius raised to the power of 4 , this effect is much less with larger diameter stems. Finally, since tapered stems are prepared by so-called line-to-line reaming and the fixation flutes cut into the bone the tapered stem design allows for safer insertion with a potentially lower risk of intraoperative fracture. With cylindrical stems, the stems are oversized relative to reaming, engage early, and require a minimum of $5 \mathrm{~cm}$ of adequate press-fit. The last few centimeters of stem insertion therefore require, on occasion, a fair bit of force, which adds to the stem design, increasing the potential risk of fracture. Although no published studies evaluate the rate of thigh pain with TFMT, Bohm and associates [3], reporting on 128 patients revised with tapered titanium stems at a mean followup of 14 years, found low rates of intraoperative fractures $(1.5 \%)$ and stress shielding of the proximal femur (3\%) compared to published complications rates for revision $\mathrm{CNCC}$ femoral stems (fracture rate 9-30\% [18, 23]; stress shielding rate $8 \%[19])$.
We therefore asked whether the TFMT femoral components are better than $\mathrm{CNCC}$ stems at achieving three important goals of revision hip arthroplasty: (1) improving quality of life; (2) avoiding complications; and (3) preserving or restoring femoral bone stock.

\section{Patients and Methods}

We used a retrospective cross-sectional cohort comparison of 219 patients with one of two femoral component designs used for revision hip arthroplasty between January 2000 and March 2006. All 34 TFMT patients from our previously published series [11] were included in this study. Many of the CNCC patients from our previous study [11] were excluded from this study because their surgical date was prior to January 2000, the date that we began using TFMT femoral stems. The indications for the use of the two stem designs evolved during the study period. Early in the study period, the CNCC stem was used if there was adequate host bone for 4 to $5 \mathrm{~cm}$ of scratch fit and the TFMT stem was reserved for cases in which this amount of scratch fit could not be obtained. Later in the study period, we increasingly used the TFMT stem despite adequate host bone. We identified 109 eligible patients who had TFMT stems (ZMR Hip System; Zimmer, Warsaw, IN). Of these, six patients had undergone revision (four implant fractures, one infection, one aseptic loosening), three had incomplete quality of life questionnaires (see below for details of the validated questionnaires employed), and five were deceased, leaving 95 patients available for quality of life outcome evaluation. We identified 115 eligible patients who had CNCC revision stems (Solution System; DePuy, Warsaw, IN). Of these, 10 patients were revised (four infections, four aseptic loosening, two implant fractures), leaving 105 patients available for quality of life outcome comparison. The minimum followup in the TFMT group was 24 months (mean, 37 months; range, 24-83 months) compared with 24 months (mean, 49 months; range, 2495 months) in the CNCC group. The mean age (70.2 years in the TFMT cohort versus 68.3 years in the CNCC cohort [p $=0.18]$ ), gender (male to female ratio of $47: 48$ for the TFMT cohort and 55:50 for the CNCC cohort), and comorbidity characterized by Charnley class were similar in the two groups $(p=0.22)$. Preoperative diagnosis was also similar for the two groups (Table $1 ; \mathrm{p}=0.12$ ). Eight patients were lost to followup in the TFMT cohort, five died, and three patients had incomplete quality of life questionnaires (see below for details of the validated questionnaires) and could not be contacted. All three patients with incomplete scores responded positively in the sections that were completed. 
Table 1. Preoperative diagnosis

\begin{tabular}{lll}
\hline $\begin{array}{l}\text { Preoperative } \\
\text { diagnosis }\end{array}$ & $\begin{array}{l}\text { TFMT cohort } \\
(\mathrm{N}=103)\end{array}$ & $\begin{array}{l}\text { CNCC cohort } \\
(\mathrm{N}=114)\end{array}$ \\
\hline Aseptic loosening & 75 & 95 \\
Infection & 17 & 9 \\
Fracture & 11 & 10 \\
\hline
\end{tabular}

TFMT $=$ tapered, fluted, modular titanium; $\mathrm{CNCC}=$ cylindrical, nonmodular cobalt chrome.

Table 2. Classification of preoperative femoral defect

\begin{tabular}{lll}
\hline $\begin{array}{l}\text { Paprosky } \\
\text { classification }\end{array}$ & $\begin{array}{l}\text { TFMT cohort } \\
(\mathrm{N}=103)\end{array}$ & $\begin{array}{l}\text { CNCC cohort } \\
(\mathrm{N}=111)\end{array}$ \\
\hline 1 & 4 & 1 \\
2 & 5 & 15 \\
$3 \mathrm{~A}^{*}$ & 29 & 60 \\
$3 \mathrm{~B}^{*}$ & 58 & 31 \\
4 & 7 & 4 \\
\hline
\end{tabular}

$* \mathrm{p}<0.0001 ; \quad$ TFMT $=$ tapered, $\quad$ fluted, modular titanium; $\mathrm{CNCC}=$ cylindrical, nonmodular cobalt chrome.

The preoperative bone defects were assessed by one author (CJR) based on the Paprosky classification [21]. The Paprosky femoral defect classification system reportedly has an interobserver $\kappa$ value of 0.42 and an intraobserver $\kappa$ value of 0.54 [12]. We identified severe defects $(\mathrm{p}<0.00001)$ in the TFMT cohort with $64 \%$ being Paprosky 3B and 4 (Table 2).

Preoperative evaluation for all patients in the study included a standard AP pelvis, crosstable lateral hip, and AP and lateral of the femur. Judet views were obtained if acetabular reconstruction was planned. All patients underwent investigations to rule out infection, including C-reactive protein, erythrocyte sedimentation rate, and complete blood count.

All patients were positioned lateral decubitus. The surgical approach used for the two cohorts was similar $(\mathrm{p}=0.13)$ with a posterolateral approach used for the majority of cases (88\% [91 of 103] for the TFMT cohort versus $80 \%$ [91 of 114] for the CNCC cohort) and the remaining patients undergoing a direct lateral approach (12\% [12 of 103] for the TFMT cohort versus 20\% [23 of 114] for the CNCC cohort). An extended trochanteric osteotomy was used more frequently for the CNCC cohort (36 of 103 for the TFMT cohort versus 72 of 114 for the CNCC cohort; $\mathrm{p}<0.001$ ). For all patients, a prophylactic cerclage wire was placed distal to the extended trochanteric osteotomy in an attempt to decrease the risk of intraoperative fracture during stem insertion.

Postoperatively, the protocol was the same for both cohorts. Patients were nonweightbearing for a period of
6 weeks, when partial weightbearing was initiated with gradual progression to full weightbearing by 3 months. For those patients who underwent a posterior approach, posterior precautions were used for the first 6 weeks, including no hip flexion beyond $90^{\circ}$ and no adduction. All patients who had an extended trochanteric osteotomy were restricted to no active abduction for the initial 6 weeks. All patients were followed at 6 weeks, 3 months, 1 year, and then yearly thereafter.

We used five validated quality of life outcome scores to assess patients: the Oxford Hip Score [6], the WOMAC Osteoarthritis Index [1], the SF-12 [22], the Hip and Knee Arthroplasty Satisfaction questionnaire [15], and the UCLA activity score. Preoperative scores, available for 66 patients $(69 \%)$ in the TFMT cohort and 58 patients $(52 \%)$ in the CNCC cohort, were similar for the two groups. The 5 validated quality of life outcome questionnaires mentioned above were mailed to patients annually during followup. Informed consent was obtained with the first request for followup quality of life information. Patients who failed to complete routine followup were sent a repeat questionnaire or contacted by telephone. We had complete postoperative data on 95 of the 109 patients (90\%) with TFMT stems and 105 of the 115 patients $(91 \%)$ with CNCC stems.

Postoperative instability was determined by a hospital chart review as well as a patient mailed questionnaire. Forty-three patients in the TFMT cohort and 38 patients in the CNCC cohort did not respond or responded partially to the quality of life questionnaires and were contacted by telephone. All patients were contacted except three patients in the TFMT cohort with incomplete questionnaires.

The occurrence of intraoperative femoral shaft fractures was documented by reviewing operative reports and early postoperative radiographs for all patients.

One of us (CJR) classified the radiographic changes in the proximal femoral bone stock as described by Bohm and Bischel [2]: A (increasing defects), B (constant defects), or $\mathrm{C}$ (osseous restoration). There are no published studies on the reliability of this classification system. However, Engh and associates [9] reported on the reliability of radiographic evaluation for femoral bone loss and noted a $\kappa$ value of 0.58 for interobserver reliability and a $\kappa$ value of 0.74 for intraobserver reliability. To minimize bias during the subjective evaluation of the radiographs, all identifying information was concealed for both the initial postoperative and most recent followup radiographs. These two films were then compared, evaluating them for a difference in the proximal femoral bone stock. If a difference was noted, the dates of the radiographs were revealed to ascertain if this represented Type A or Type C. Fixation of cementless femoral components for both cohorts were evaluated radiographically according to the criteria of Engh and associates [10]. 
We determined differences in WOMAC pain and stiffness, Oxford Hip Score, and overall satisfaction between the TFMT and CNCC cohorts using two-sample t-tests. A separate analysis was completed comparing outcomes scores for those patients with a preoperative diagnosis of aseptic loosening. A difference in the occurrence of intraoperative fractures was also noted between the two cohorts using a two-tailed z-test for proportions. We performed another analysis to ascertain if the use of an extended trochanteric osteotomy influenced the occurrence of intraoperative fractures. An exact chi square, used for analysis of radiographically evident changes to the proximal femoral host bone stock, demonstrated a difference between the two cohorts.

\section{Results}

Despite the TFMT stems being used in cases with worse bone loss, all quality of life outcome scores were higher in the TFMT cohort (Table 3): WOMAC pain $(\mathrm{p}=0.04)$ and stiffness scores $(p=0.009)$, Oxford Hip $(p=0.008)$, and overall satisfaction $(\mathrm{p}=0.04)$. Among the patients with aseptic loosening, we found higher outcome scores for the TFMT cohort for WOMAC stiffness $(\mathrm{p}=0.042)$, Oxford Hip $(\mathrm{p}=0.032)$, and satisfaction recreation $(\mathrm{p}=0.036)$.

There were more $(\mathrm{p}=0.002)$ intraoperative fractures observed for the CNCC cohort (29 of 114) compared with the TFMT group (nine of 103). We observed no difference $(\mathrm{p}=0.641)$ in the occurrence of postoperative instability between the two groups (eight of 103 for the TFMT cohort versus 12 of 114 for the CNCC cohort). We found a similar percentage $(p=0.94)$ of fractures for patients regardless of whether an extended trochanteric osteotomy was used ( 19 of 72 or $26 \%$ with the extended trochanteric osteotomy patients versus 10 of 42 or $24 \%$ for the nonextended

Table 3. Quality of life outcome scores

\begin{tabular}{llll}
\hline $\begin{array}{l}\text { Quality of life } \\
\text { measure }\end{array}$ & $\begin{array}{l}\text { TFMT stems } \\
(\mathrm{N}=95)\end{array}$ & $\begin{array}{l}\text { CNCC stems } \\
(\mathrm{N}=105)\end{array}$ & $\mathrm{p}$ Value \\
\hline Oxford hip score & 77 & 69 & 0.008 \\
SF-12 mental component & 53 & 51 & 0.11 \\
SF-12 physical component & 39 & 38 & 0.39 \\
Satisfaction overall score & 90 & 81 & 0.04 \\
WOMAC pain & 84 & 78 & 0.04 \\
WOMAC stiffness & 76 & 68 & 0.009 \\
UCLA activity score & 5.1 & 4.6 & 0.11 \\
\hline
\end{tabular}

Oxford Hip score and WOMAC scores are normalized to a range of 0 to 100 with 0 being worst and 100 being best; TFMT $=$ tapered, fluted, modular titanium; $\mathrm{CNCC}=$ cylindrical, nonmodular cobalt chrome.
Table 4. Radiographically evident changes to the proximal femur host bone stock

\begin{tabular}{lll}
\hline $\begin{array}{l}\text { Changes to the proximal femur } \\
\text { host bone stock }\end{array}$ & $\begin{array}{l}\text { TFMT cohort } \\
(\mathrm{N}=79)\end{array}$ & $\begin{array}{l}\text { CNCC cohort } \\
(\mathrm{N}=62)\end{array}$ \\
\hline Type A: bone loss & 10 & 26 \\
Type B: no change & 37 & 31 \\
Type C: bone restoration & 32 & 5 \\
\hline
\end{tabular}

TFMT $=$ tapered, fluted, modular titanium; $\mathrm{CNCC}=$ cylindrical, nonmodular cobalt chrome.

trochanteric osteotomy patients).There was no major difference noted in postoperative instability.

More patients in the TFMT cohort had an increase $(p<0.0001)$ in the percentage of patients with osseous restoration (Type $\mathrm{C}$ ) and a decrease $(\mathrm{p}=0.02)$ in the percentage with increasing defects (Type A) compared with the CNCC cohort (Table 4). All surviving implants in the CNCC cohort were well fixed. All surviving implants except one in the TFMT cohort were well fixed. This patient has subsequently been revised and is included in the failure group for the TFMT cohort.

\section{Discussion}

Reports of early and midterm survivorship for TFMT stem designs demonstrate equivalent results when compared with CNCC femoral components, ranging from $86 \%$ to $99 \%[2,3,8,14,17,19,20,23]$. In a previous report from this institution in a smaller cohort, it was demonstrated that the TFMT femoral components had advantages in terms of patient-reported quality of life [11]. We attempted to see if this difference was still valid in a larger cohort followed for a longer period of time. In addition, we asked whether there were differences in these two stem designs in terms of intraoperative fractures, postoperative instability, and preservation or restoration of host bone stock.

There are several limitations of this study consistent with the limitations of a cross-sectional study design. First, preoperative quality of life outcome scores are the most important predictor of postoperative outcomes [7]. The lack of preoperative scores in all patients is a potential confounder. Preoperative scores were available for $69 \%$ (65 of 95) of patients in the TFMT cohort and 52\% (55 of $105)$ of patients in the CNCC cohort. However, the reasons for not having scores on all patients were random. The patients in the CNCC arm were seen in the preadmission clinic before the institution of routine data collection for quality of life on these patients. There was no selection bias in either group in terms of who filled out preoperative questionnaires. Patients who were missed were essentially a random event. Given this fact and that the preoperative 
scores available would be a negative confounder for the TFMT (lower overall preoperative scores), the authors do not believe this affected the conclusions on quality of life of the study. Second, we recognize the selection bias in the study. Early in the study period, the TFMT stems were used for more severe defects when it was believed 4 to $5 \mathrm{~cm}$ of scratch fit with a cylindrical porous-coated stem could not be attained. This bias was confirmed with the radiographic review, which revealed worse preoperative defects in the TFMT stem cohort. Although this bias was definitely present, if anything, it should be a bias against success for the TFMT cohort.

The quality of life outcome measures used for this study include the generic SF-12, the disease-specific WOMAC Osteoarthritis Index, the joint-specific Oxford Hip Score as well as the Satisfaction and UCLA activity scores. Although there is substantial overlap, each of these tools focuses on a different aspect of patient outcome. The combination of these various tools was used to increase the overall sensitivity to subtle clinical differences. Our data suggest there is a difference in quality of life outcome scores at 2 years favoring the TFMT group despite worse preoperative bone defects. In those patients operated for aseptic loosening we also observed better scores in the TFMT cohort. The improved outcomes may be related to a decrease in modulus mismatch between the implant and host bone as well as enhanced hip biomechanics. We believe further studies evaluating for thigh pain or improvements in hip biomechanics may be warranted to better explain the improvement in quality of life scores observed.

There were four implant fractures in the TFMT cohort compared with two in the CNCC cohort. Although concerning, all stem fractures occurred at the modular junction in an older design resulting from the lack of bone support of the modular junction. The modular junction has been redesigned with improved strength at the junction to reduce or eliminate stem fracture. Stem fractures have not been observed with the new design. The TFMT cohort had fewer intraoperative cortical femur split fractures. This reduced incidence is likely the result of the tapered stem geometry, which allows rigid fixation with reduced insertion force compared with that commonly required for the cylindrical design. While we used an extended trochanteric osteotomy more commonly in the CNCC cohort we found no difference in the likelihood of fracture in the two groups.

The subjective radiographic review in this study suggests the TFMT cohort had better maintenance and restoration of the proximal femur at a minimum 2-year followup. Radiographic evaluation is not the gold standard for evaluating bone loss and the definitive answer to the question of proximal femoral stress shielding would require early postoperative and long-term followup dual-energy xray absorptiometry scans [9].

One downside of the modular tapered titanium stem is the risk of fracture at the modular junction. There were four implant fractures in the TFMT cohort compared with two in the CNCC cohort. Although concerning, all stem fractures occurred at the modular junction in an older design, which is no longer in use. The construct has been redesigned with improved geometry at the junction to reduce or eliminate stem fracture. Stem fractures have not been observed with the new design.

Our short to midterm data suggest TFMT revision components are superior to CNCC stems at achieving three of the four main goals of revision femoral surgery: improved patient quality of life, decreased complications, and restoration of the proximal femur. We believe our observations, in addition to other reported early and midterm successful results, suggest these new designs are an attractive alternative to other cementless designs.

Acknowledgments We thank Dr Nelson V. Greidanus for recruiting subjects in this study.

\section{References}

1. Bellamy N, Kirwan J, Boers M, Brooks P, Strand V, Tugwell P, Altman R, Brandt K, Dougados M, Lequesne M. Recommendations for a core set of outcome measures for future phase III clinical trials in knee, hip, and hand osteoarthritis. Consensus development at OMERACT III. J Rheumatol. 1997;24:799-802.

2. Bohm P, Bischel O. Femoral revision with the Wagner SL revision stem: evaluation of one hundred and twenty-nine revisions followed for a mean of 4.8 years. J Bone Joint Surg Am. 2001;83:1023-1031.

3. Bohm P, Bischel O. The use of tapered stems for femoral revision surgery. Clin Orthop Relat Res. 2004;420:148-159.

4. Cameron HU. The long-term success of modular proximal fixation stems in revision total hip arthroplasty. J Arthroplasty. 2002;17(4 Suppl 1):138-141.

5. Christie MJ, DeBoer DK, Tingstad EM, Capps M, Brinson MF, Trick LW. Clinical experience with a modular noncemented femoral component in revision total hip arthroplasty: 4- to 7-year results. J Arthroplasty. 2000;15:840-848.

6. Dawson J, Fitzpatrick R, Carr A, Murray D. Questionnaire on the perceptions of patients about total hip replacement. J Bone Joint Surg Br. 1996;78:185-190.

7. Dawson J, Fitzpatrick R, Murray D, Carr A. Comparison of measures to assess outcomes in total hip replacement surgery. Qual Health Care. 1996;5:81-88.

8. Engh CA, Jr., Hopper RH, Jr., Engh CA, Sr. Distal ingrowth components. Clin Orthop Relat Res. 2004;420:135-141.

9. Engh CA, Jr., McAuley JP, Sychterz CJ, Sacco ME, Engh CA, Sr. The accuracy and reproducibility of radiographic assessment of stress-shielding. A postmortem analysis. J Bone Joint Surg Am. 2000;82:1414-1420.

10. Engh CA, Massin P, Suthers KE. Roentgenographic assessment of the biologic fixation of porous-surfaced femoral components. Clin Orthop Relat Res. 1990;257:107-128. 
11. Garbuz DS, Toms A, Masri BA, Duncan CP. Improved outcome in femoral revision arthroplasty with tapered fluted modular titanium stems. Clin Orthop Relat Res. 2006;453:199202.

12. Gozzard C, Blom A, Taylor A, Smith E, Learmonth I. A comparison of the reliability and validity of bone stock loss classification systems used for revision hip surgery. J Arthroplasty. 2003;18:638-642.

13. Hamilton WG, Cashen DV, Ho H, Hopper RH, Jr., Engh CA. Extensively porous-coated stems for femoral revision: a choice for all seasons. J Arthroplasty. 2007;22(4 Suppl 1):106-110.

14. Kang MN, Huddleston JI, Hwang K, Imrie S, Goodman SB. Early outcome of a modular femoral component in revision total hip arthroplasty. J Arthroplasty. 2008;23:220-225.

15. Katz JN, Phillips CB, Baron JA, Fossel AH, Mahomed NN, Barrett J, Lingard EA, Harris WH, Poss R, Lew RA, Guadagnoli E, Wright EA, Losina E. Association of hospital and surgeon volume of total hip replacement with functional status and satisfaction three years following surgery. Arthritis Rheum. 2003;48:560-568.

16. Krishnamurthy AB, MacDonald SJ, Paprosky WG. 5- to 13-year follow-up study on cementless femoral components in revision surgery. J Arthroplasty. 1997;12:839-847.
17. McInnis DP, Horne G, Devane PA. Femoral revision with a fluted, tapered, modular stem seventy patients followed for a mean of 3.9 years. J Arthroplasty. 2006;21:372-380.

18. Meek RM, Garbuz DS, Masri BA, Greidanus NV, Duncan CP. Intraoperative fracture of the femur in revision total hip arthroplasty with a diaphyseal fitting stem. J Bone Joint Surg Am. 2004;86:480-485.

19. Moreland JR, Bernstein ML. Femoral revision hip arthroplasty with uncemented, porous-coated stems. Clin Orthop Relat Res. 1995;319:141-150.

20. Park YS, Moon YW, Lim SJ. Revision total hip arthroplasty using a fluted and tapered modular distal fixation stem with and without extended trochanteric osteotomy. $J$ Arthroplasty. 2007;22:993-999.

21. Sporer SM, Paprosky WG. Revision total hip arthroplasty: the limits of fully coated stems. Clin Orthop Relat Res. 2003;417:203-209.

22. Ware J, Jr., Kosinski M, Keller SD. A 12-Item Short-Form Health Survey: construction of scales and preliminary tests of reliability and validity. Med Care. 1996;34:220-233.

23. Weeden SH, Paprosky WG. Minimal 11-year follow-up of extensively porous-coated stems in femoral revision total hip arthroplasty. J Arthroplasty. 2002;17(4 Suppl 1):134-137. 